\title{
Enfermedad de Gaucher asociado a linfoma de celulas T tipo hidroa vacciniforme like: reporte de un caso
}

\section{Gaucher disease associated with T-cell lymphoma hydroa vacciniforme like: Case report}

Correspondencia

Junior Julio Zapata Choque zapatajunior057@gmail.com

\section{Recibido: 05/11/2018}

Arbitrado por pares

Aprobado: 17/04/2019

Citar como: Zapata JJ, Nina G, Orccosupa DJ, Urrutia K. Enfermedad de Gaucher asociado a linfoma de celulas $T$ tipo hidroa vacciniforme like: reporte de un caso. Acta Med Peru. 2019;36(2):129-33
Junior J. Zapata1,a, Gary Nina, ${ }^{1, a}$, David J. Orccosupa ${ }^{1, a}$, Katya Urrutiaa,b,c

1 Asociación Científica de Estudiantes de Medicina de la Universidad Andina del Cusco. Cusco, Perú.

2 Servicio de hematología clínica, Hospital Nacional Adolfo Guevara Velazco. Cusco, Perú.

a Estudiantes de Medicina; b médico cirujano; c especialista en Hematología Clínica

\section{RESUMEN}

La enfermedad de Gaucher es un trastorno metabólico autosómico recesivo crónico y progresivo que se caracteriza por depósito lisosomal con deficiencia de la enzima glucocerebrosidasa ácida produciendo causando daño celular y disfunción orgánica; se asocia a enfermedades neoplásicas hematológicas; sin embargo, su asociación con linfomas es rara. El linfoma hidroa vacciniforme like es una enfermedad rara per se pero afecta en más casos a niños y adolescentes; está caracterizado por lesiones vesiculares cutáneas, adenopatías y visceromegalias. Presentamos el caso de una niña proveniente de una comunidad andina de Cusco de 12 años que presentó durante siete años vesículas costrosas, fiebre, edema facial con ulcera palpebral, ganglios palpables, hepatoesplenomegalia, acompañado de pancitopenia. Se realizó un estudio enzimático y genético observándose deficiencia de $\beta$-glucosidasa y del gen GBA; en la biopsia de piel se encontró un infiltrado linfoide dérmico con pleomorfismo nuclear compatible con linfoma de células T tipo hidroavacciniforme like, posteriormente la paciente presentó leve mejoría con el tratamiento de reemplazo enzimático pero falleció debido al shock hipovolémico tras dos episodios de hemorragia digestiva baja.

Palabras clave: Enfermedad de Gaucher; Linfoma no Hodgkin; Glucocerebrosidasa; Malignidades hematológicas (fuente: DeCS BIREME). 


\begin{abstract}
Gaucher disease is a chronic and progressive autosomal recessive metabolic disorder that is characterized by lysosome depots with deficiency of acid glucocerebrosidase enzyme, which leads to cell damage and organic dysfunction. This condition is associated with some hematological malignancies; however, its association with lymphomas is rare. Hydroa vacciniform-like lymphoma is a rare condition per se, but it is becoming increasingly frequent in children and adolescents. It is characterized by the presence of cutaneous vesicular lesions, adenopathy, and visceromegaly. We present the case of a 12-year old girl from an Andean community in Cusco who presented with crusting vesicles, fever, face edema with eyelid ulceration, palpable lymph nodes, and hepatosplenomegaly, accompanied by pancytopenia. An enzymatic and genetic study was carried out, and both $\beta$-glucosidase deficiency and GBA gene deficiency were found. Skin biopsies revealed a dermal lymphoid infiltrate with nuclear pleomorphism compatible with hydroa vacciniform like T-cell lymphoma. Subsequently, the patient developed slight improvement with the enzyme replacement therapy, but she died because of hypovolemic shock after two episodes of low gastrointestinal hemorrhage.
\end{abstract}

Keywords: Gaucher disease; Lymphoma, non-Hodgkin; Glucocerebrosidase; Hematologic neoplasms (source: MeSH NLM).

\section{INTRODUCCIÓN}

La enfermedad de Gaucher (EG) es una deficiencia de la enzima glucocerebrosidasa acida que produce la acumulación de glucocerebrósidos en las células de la médula ósea, el bazo, el hígado, los pulmones, tejido esquelético y neuronas causando disfunción orgánica ${ }^{[1]}$

El hidroa vacciniforme es una fotodermatosis de etiología desconocida que se puede presentar en forma de lesiones ubicadas solo en lugares fotoexpuestos, que va desapareciendo con la edad y que en la adolescencia llega a atenuarse sin comprometer el estado general; sin embargo, su semejanza con la variante maligna llamada linfoma cutáneo de células T tipo hidroa vacciniforme like (LHVL), puede retrasar su diagnóstico. En esta forma de linfoma las lesiones eritematosas vesiculares se observan en zonas no expuestas y expuestas a la luz (rayos UV) perjudicando a la vez el estado general del paciente ${ }^{[2,3]}$.

La EG se asocia a neoplasias, en la mayoría de los casos los del tipo hematológico como el linfoma Hodking y no Hodking de células $B$ juntamente con leucemias mieloides agudas cuya incidencia y prevalencia es mayor en niños; además, vemos que la EG es un factor de riesgo para desarrollar ganmapatías monoclonales tipo mieloma múltiple o amiloidosis además de poder transformar las ganmapatías monoclonales de significado incierto a mieloma multiple ${ }^{[4]}$. La asociación con linfomas de células $T$ es rara y dentro de ellas la asociación con linfomas cutáneos de células T no está descrita.

Presentamos el caso de una niña con enfermedad de Gaucher y linfoma cutáneo de células T tipo hidroa vacciniforme like.

\section{REPORTE DE CASO}

Paciente mujer de 12 años de edad de etnia mestiza procedente de una comunidad de Cusco, de medio socioeconómico bajo, con antecedentes de un adecuado desarrollo psicomotor, que de manera esporádica durante siete años presentó vesículas costrosas de centro necrótico con supuración intermitente ubicados en cara, nariz, manos, tórax y muslos, acompañadas de edema bipalpebral (Figura 1), con remisión parcial al uso de antibióticos tópicos y sistémicos; además, adenomegalias palpables a nivel retroauricular con hepatomegalia palpable.

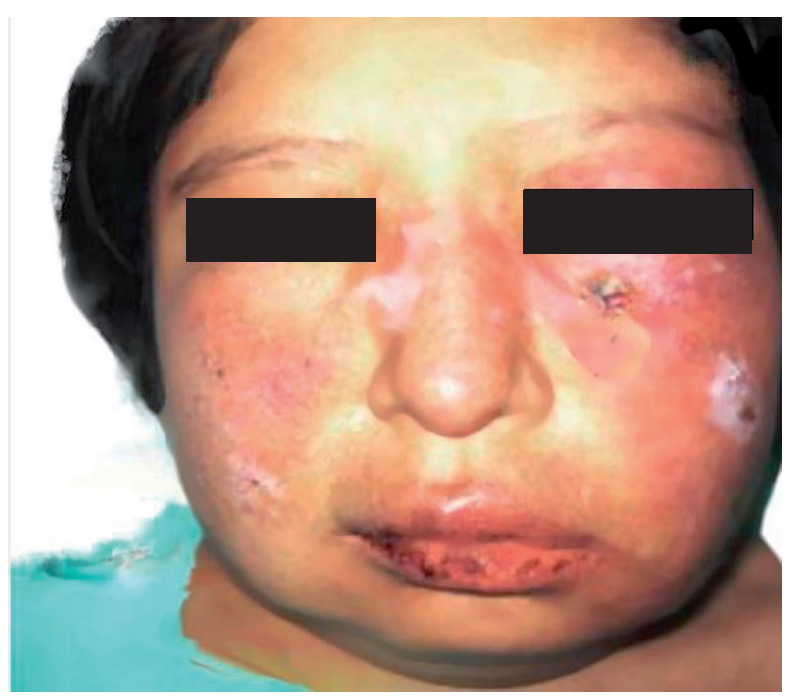

Figura 1. Paciente mujer con lesiones costrosas de centro necrótico con supuración intermitente ubicados en cara y nariz.

Acudió al servicio de urgencias por presentar un cuadro clínico de cinco meses de duración caracterizado por incapacidad progresiva para marchar, dificultad para mantenerse de pie y disminución de la fuerza en ambos miembros inferiores, llegando al uso de silla de ruedas; diez días antes de su ingreso presentó dolor abdominal intenso en fosa iliaca derecha acompañado de náuseas y vómitos.

Al examen físico, se observó a una paciente en mal estado de nutrición, con leve retraso en el crecimiento. Se hallaron lesiones vesiculo-costrosas en cara, brazos, manos y muslos, con adenomegalia palpable a nivel cervical e inguinal, conjuntivas pálidas, abdomen blando y doloroso a nivel de la fosa iliaca derecha con una hepatoesplenomegalia palpable. 
Los resultados del estudio de laboratorio mostraron leucocitos: 2,41 millones $/ \mathrm{mm}^{3}$, neutrófilos: $64 \%$, linfocitos: $33 \%$, monocitos: $3 \%$, eritrocitos: 4,34 millones $/ \mathrm{mm}^{3}$, hemoglobina $(\mathrm{HB}): 12,2$ $\mathrm{mg} / \mathrm{dL}$, hematocrito (HCT): $38,5 \%$, volumen corpuscular medio (VCM): 77,6 fL, hemoglobina corpuscular media (HCM): $24,8 \mathrm{pg} /$ $\mathrm{dL}$, plaquetas: $59000 / \mathrm{mm} 3$, albumina: $1,4 \mathrm{~g} / \mathrm{dL}$, globulinas: 2,7 $\mathrm{g} / \mathrm{dL}$, bilirrubina total: $3,1 \mathrm{~g} / \mathrm{dL}$, bilirrubina directa: $2,74 \mathrm{~g} / \mathrm{dL}$, aspartato aminotransferasa: $320 \mathrm{UI} / \mathrm{L}$, alanina aminotrasferasa: $267 \mathrm{UI} / \mathrm{L}$, fosfatasa alcalina: $1630 \mathrm{UI} / \mathrm{L}$.

El ultrasonido abdominal reveló hepatoesplenomegalia con ecogenicidad normal. Se le solicitó cultivo de secreción de las lesiones vesiculares aislándose Staphyloccocus aureus, por lo que se inició el tratamiento intravenoso con oxacilina, sin mostrar mejoría en el tiempo.

En los días posteriores en la analítica mostró HB: $11,3 \mathrm{mg} / \mathrm{dL}$, HCT: 34,2\%, VCM: 67,3 fL, HCM: 22,2 pg/dL, plaquetas: 55000/ $\mathrm{mm}^{3}$, ANA (-), ANCA (-), Anti-DNA (-), gen Eber (+) para el virus Epstein Barr, biopsia de piel en zonas afectadas y no afectadas; además de biopsia de médula ósea.

En la biopsia de medula ósea se observó macrófagos de citoplasma amplio y claro cargados de lípidos. En la biopsia de piel intacta, se encontró la presencia de un infiltrado crónico de células de citoplasma amplio y claro; a esta muestra se le realizó estudio inmunohistoquimico que mostró una elevación de CD68, diagnosticándose una enfermedad de depósito. Las muestras de la biopsia de piel, medula ósea y sangre periférica mostraron deficiencia de la enzima B-glucosidasa $(0,65 \mathrm{nmol} / \mathrm{h} /$ mg; valor normal [VN]: 3-15,8), además se hizo un estudio genético encontrando deficiencia del gen GBA (3,33 pmol/punch/h; $\mathrm{VN}: \geq 7)$, con mutación en el intron N370S; diagnosticándose EG de tipo 1.

En la biopsia de piel afectada, se visualizó infiltrado linfoide atípico que comprometió dermis superficial y profunda, con marcado pleomorfismo nuclear y focos de necrosis, hallazgos consistentes con linfoma no Hodgkin (Figura 2); además, reveló $\mathrm{CD} 3(+), \mathrm{CD} 8(+), \mathrm{CD} 30(+)$ en células grandes, CD4 (+) con células $T$ reactivas, compatible con linfoma de células $T$ tipo hidroa vacciniforme like (LHVL) (Figura 2).

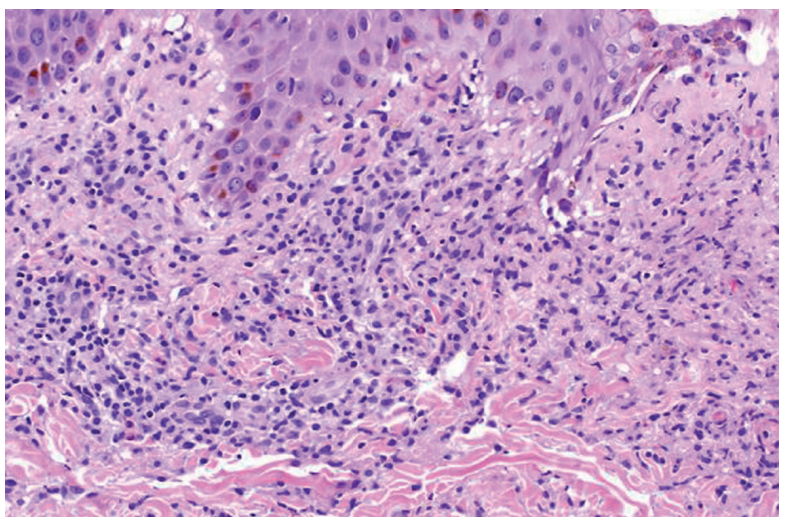

Figura 2. Denso infiltrado de linfocitos atípicos en dermis y tejido celular subcutáneo Inmunomarcación positiva para CD3, CD4 y/o CD8 (50x).
En el proteinograma sérico se hallaron proteínas totales $8,94 \mathrm{~g} /$ $\mathrm{dL}$ (VN: 5,80-8,10 g/dL), relación albumina /globulina 0,74 (VN: 1,30-2,50), albumina $3.80 \mathrm{~g} / \mathrm{dL}$ (VN: 3,50-5,20 g/dL), $\alpha-1$ globulina $0,43 \mathrm{~g} / \mathrm{dL}$ (VN: 0,15-0,40 g/dL), gamma-globulina: $2,77 \mathrm{~g} / \mathrm{dl}$ (VN: $0.60-1,60 \mathrm{~g} / \mathrm{dl})$; dando a conocer una hipergammaglobulinemia policlonal, la cual se confirmó con inmunofijación (aumento de $\operatorname{lgA}, \lg \mathrm{g})$. La densitometría ósea reveló osteoporosis asintomática en cadera.

Se decidió iniciar tratamiento de reemplazo enzimático con imiglucerasa $60 \mathrm{UI} / \mathrm{kg}$ cada dos semanas como dosis inicial por seis meses; posteriormente se pasó a $15 \mathrm{UI} / \mathrm{kg}$ por un año, según esquema de consolidación. Para el linfoma cutáneo la paciente no toleró el esquema $\mathrm{CHOP}$, por lo que se inició tratamiento con talidomida y betametasona, las cuales no demostraron una mejoría significativa en las lesiones cutáneas.

En las evaluaciones posteriores se apreció mejoría gradual de la paciente con regresión de la hepatoesplenomegalia, mejoría de la trombocitopenia y fuerza muscular, por lo que se decidió continuar con el tratamiento de reemplazo enzimático por 18 meses. Durante su evolución la paciente presentó bruscamente hematoquecia, lo que motivó el ingreso por emergencia; en dicho servicio se identificaron mucosas pálidas, dolor abdominal, con HB $7 \mathrm{mg} / \mathrm{dL}$, plaquetas: $7000 / \mathrm{mm}^{3}$, tiempo de protrombina $20 \mathrm{~s}$, INR 4 y tiempo de tromboplastina parcial activado $80 \mathrm{~s}$.

Mientras estuvo en emergencia, presentó un episodio más de hematoquecia. Se le realizó una endoscopia que dio el diagnóstico de esofagitis crónica, prolapso cardial y gastritis crónica; en la colonoscopía se evidenció una colitis erosiva con hemorragia leve. Posteriormente volvió a presentar otro episodio de hematoquecia complicándose con shock hipovolémico requiriendo soporte transfusional y medidas de resucitación con uso de ventilación mecánica y vasopresores. A pesar de los cuidados la paciente falleció.

\section{DISCUSIÓN}

El linfoma cutáneo tipo hidroa vacciniforme like es una neoplasia rara y agresiva que afecta a personas jóvenes de Asia y de América Latina ${ }^{[2]}$. Esta neoplasia es un linfoma primario de células T/NK infrecuente que suele presentarse en individuos de piel blanca, y que hasta en un $25 \%$ de los casos existe antecedente familiar de fotosensibilidad; es de mal pronóstico y presenta pocas probabilidades de sobrevida ${ }^{[3]}$. En el caso de nuestra paciente al ser propia de una comunidad andina del Cusco comparte la epidemiologia de América Latina; no obstante, los linfomas cutáneos suelen ser más frecuentes en adultos -la incidencia en niños es de 0,3 por cada 1 millón de habitantes- ${ }^{[5]}$.

Su origen está relacionado fuertemente al virus Epstein Barr (VEB) ${ }^{[2]}$. En el estudio publicado por Liu ${ }^{[6]}$ el trastorno linfoproliferativo tipo hidroa vacciniforme (HVLLPD) en pacientes chinos mostró un comportamiento indolente y crónico en la mayoría de los casos, curso que difiere de los casos con HVLLPD en Latinoamérica que tienen un pobre pronóstico y son raros en niños; además, 
los pacientes con altas cargas séricas de ADN del EBV tenían un mayor riesgo de evolucionar hacia una enfermedad agresiva.

Dentro de las anormalidades genéticas que causa el VEB, la estimulación antigénica crónica juega un rol importante en la generación de linfomas no Hodking ${ }^{[7]}$, por lo que constituye un factor de riesgo para desarrollar linfoma de cualquier índole. En nuestra paciente fue positiva la presencia del VEB.

El examen físico en pacientes con linfoma cutáneo revela erupciones recurrentes con vesículas ampollosas y supurativas ${ }^{[8]}$. En nuestra paciente las vesículas costrosas se ubicaron en cara, cuello, ambas manos, tronco y muslos; similar a los hallazgos que hizo Beltrán en Perú, cuyo reporte describió lesiones en áreas no fotoexpuestas ${ }^{[9]}$; diferente a lo que halló Plaza, quien identificó lesiones que se limitaron a cara y cuello ${ }^{[8]}$; de igual manera Montalvo describió lesiones cutaneas en áreas fotoexpuestas como heridas, pápulas, vesículas, costras y cicatrices ${ }^{[10]}$.

La anatomía patológica del LHVL muestra un infiltrado denso de linfocitos atípicos en dermis y tejido celular subcutáneo, con marcadores positivos de inmunofijacion para CD3, CD4 y/o CD8; además, los casos de LHVL muestran múltiples inmunofenotipos ${ }^{[5]}$. Algunos casos presentaron un fenotipo de células T con expresión de CD4, CD8, CD45, y otros con un marcador CD56 para células NK CD56 ${ }^{[11]}$. En nuestro caso, las células tumorales exhibieron CD3 $(+), \operatorname{CD} 8(+), \operatorname{CD} 30(+)$ en células grandes, CD4 (+) con células T reactivas. Quintanilla realizó la prueba de marcador citotóxico TIA 1 resultando positivos en todos sus casos ${ }^{[12]}$. No se realizó dicha prueba a nuestra paciente por falta de disponibilidad de la prueba.

La EG es un trastorno de almacenamiento lisosomal, debido a la deficiencia hereditaria del gen GBA que codifica a la enzima glucocerebrosidasa acida ${ }^{[13]}$. Las mutaciones en el gen GBA más comunes en el mundo son N370S y L444P, representando entre el 50 y el $62 \%$ de las mutaciones en todas las poblaciones, excepto entre los Ashkenazi, que representan el 93\% de los alelos mutantes ${ }^{[14]}$. En nuestra paciente observamos la mutación en el intron N370S, lo cual se encuentra dentro de lo más frecuentes; adicionalmente, ella procedía de una región de los andes peruanos de etnia mestiza, sin embargo, la EG tipo 1 es más frecuente en personas de descendencia judía Ashkenazi ${ }^{[15]}$.

Las manifestaciones clínicas en la EG tipo 1 son: dolor óseo, osteopenia, anemia, trombocitopenia, hepatoesplenomegalia y discapacidad a largo plazo, siendo la pancitopenia y la hepatoesplenomegalia parte de las manifestaciones clínicas del LHVL ${ }^{[16]}$. En nuestra paciente se pudo observar el desarrollo de osteopenia en cadera, además de una disminución de la fuerza en miembros inferiores de manera progresiva. El diagnóstico definitivo se establece demostrando la disminución en la actividad de la enzima B-glucocerebrosidasa ${ }^{[17]}$; tal como ocurrió en nuestro caso, por un análisis de actividad enzimática en sangre periférica.

Los pacientes con EG tienen un riesgo significativo de desarrollar 14,7 veces más un cáncer hematológico ${ }^{[18]}$. La mayoría de las neoplasias que se asocian a EG son las leucemias, mielomas y linfomas Hodking y no Hodking asociados a células $B^{[18]}$, lo que convierte a nuestra paciente en un caso inédito de $E G$ asociado a LHVL. La asociación entre EG y LHVL no está bien entendida; sin embargo, se describieron factores que podrían contribuir al desarrollo del cáncer en EG, incluyendo la inflamación crónica, polarización aberrante de los macrófagos, la esplenectomía y la disfunción lisosomal ${ }^{[19]}$.

En cuanto al tratamiento de la EG, es común el reemplazo enzimático con imiglucerasa, valeglucerasa y el eliglustat que se usa en pacientes con sintomatología significativa. Dentro de la terapia debe estar el manejo de las afecciones asociadas como infecciones y pancitopenia ${ }^{[20]}$. El objetivo del tratamiento es la resolución de la pancitopenia, hepatoesplenomegalia y mejoría del estado general sobre todo en el aspecto motor [21]; estos cambios no se resolvieron totalmente en nuestro paciente, por lo que la pancitopenia y la hepatomegalia eran secundarios al LHVL.

En el presente caso clínico la paciente recibió para la EG, imiglucerasa siguiendo un protocolo de tratamiento durante 18 meses logrando una evolución favorable. En cuanto al tratamiento del LHVL, algunos autores proponen como tratamiento el esquema CHOP ${ }^{[5]}$. Sin embargo, nuestro paciente no recibió el esquema de elección sino talidomida y betametasona. Quintanilla reportó una parcial remisión de las lesiones cutáneas mejorando el cuadro clínico con el uso de corticoides y talidomida ${ }^{[12]}$. Por el contrario, nuestra paciente no mostró mejoría significativa.

En cuanto al desenlace del linfoma cutáneo, una serie paraguaya reflejó una mortalidad de $50 \%$, sobretodo en casos con compromiso extracutáneo como hepatoesplenomegalia - linfadenopatía regional ${ }^{[3]}$. Nuestra paciente también fue influenciada por los efectos sistémicos de la EG por lo que al presentar plaquetopenia también tuvo compromiso de los factores de coagulación (prolongación de los tiempos de coagulación), hallazgos comparables con el estudio de Hugues, quien vio que las complicaciones hematológicas no solo fueron la disminución de plaquetas sino la prolongación de factores de coagulación asociándose a mayor mortalidad ${ }^{[22]}$. La mortalidad de la EG tipo 1 se produce en forma variable sin tiempo definido ${ }^{[23]}$.

Concluimos, que la presentación de LHVL asociado a EG es una condición de mal pronóstico y un reto en cuanto al manejo. Siendo este el primer caso reportado de tal asociación queda muchas incógnitas aún por resolver.

Agradecimientos: al Dr. Charles Augusto Huamani Saldaña, por la revisión crítica del manuscrito.

Contribución de los autores: Los cuatro autores han contribuido en el diseño, redacción, revisión critica y aprobación del manuscrito a ser publicado.

\section{Fuentes de financiamiento: Autofinanciado}

Conflictos de interés: los autores declaran no tener conflictos de interés. 


\section{REFERENCIAS BIBLIOGRÁFICAS}

1. Colquicocha-Murillo M, Cucho-Jurado J, Eyzaguirre-Zapata RM, Manassero-Morales8 G, Moreno-Larrea M del C, Salas-Arbizu $\mathrm{KL}$, et al. Guía para diagnóstico y tratamiento de la Enfermedad de Gaucher. Rev Med Hered. 2015;26(2):103-21.

2. Durán McKinster C, Maldonado RR. Hidroa vacciniforme-like o enfermedad de Ruiz Maldonado. Dermatología Rev Mex. 2013;57(6):464-7.

3. Espínola Espínola RB, Salinas AR, Guzmán Fawcet AJ, Mendoza de Sánchez MG, Celías LF, Samudio Domínguez GC. Hidroa vacciniforme type lymphoma in Pediatrics. 2015;7(1):37-40.

4. Arends M, Van Dussen L, Biegstraaten M, Hollak CEM. Malignancies and monoclonal gammopathy in Gaucher disease; a systematic review of the literature. $\mathrm{Br} J$ Haematol. 2013;161(6):832-42.

5. Fernández MF, Cervini AB, Chantada G, Pierini AM. Linfoma cutáneo de células T hidroa vacciniforme-like. Arch Argent Dermatol. 2013;63(6):219-25.

6. Liu Y, Ma C, Wang G, Wang L. Hydroa vacciniforme-like lymphoproliferative disorder: Clinicopathologic study of 41 cases. J Am Acad Dermatol. 2019;81(2):534-40.

7. Beltramino M, Calmet R, Gatica M. Virus de Epstein-Barr y su relación con el desarrollo de enfermedades linfoproliferativas. Hematología. 2005;9(2)39-54.

8. Plaza JA, Sangueza M. Hydroa vacciniforme-like lymphoma with primarily periorbital swelling: 7 cases of an atypical clinical manifestation of this rare cutaneous T-cell lymphoma. Am J Dermatopathol. 2015;37(1):20-5.

9. Beltrán BE, Maza I, Moisés-Alfaro CB, Vasquez L, Quiñones $P$, Morales $D$, et al. Thalidomide for the treatment of hydroa vacciniforme-like lymphoma: report of four pediatric cases from Peru. Am J Hematol. 2014;89(12):1160-1.

10. Montalvo N, Redroban L. Hydroa Vacciniforme-Like EBV-Positive Cutaneous T-Cell Lymphoma, First Report of 2 Cases in Ecuador. Am J Dermatopathol. 2016;38(5):e57-9.

11. Li Y, Chen X, Tian X, Li B, Li Z. Primary cutaneous hydroa vacciniforme-like lymphoma with indolent clinical course: report of two cases and review of literature. Int J Surg Pathol. 2013;21(2):161-8.
12. Quintanilla-Martinez L, Ridaura C, Nagl F, Sáez-de-Ocariz M, Durán-McKinster C, Ruiz-Maldonado R, et al. Hydroa vacciniforme-like lymphoma: a chronic EBV+ lymphoproliferative disorder with risk to develop a systemic lymphoma. Blood. 2013;122(18):3101-10.

13. Baris HN, Cohen IJ, Mistry PK. Gaucher disease: the metabolic defect, pathophysiology, phenotypes and natural history. Pediatr Endocrinol Rev. 2014;12 Suppl 1:72-81.

14. Koprivica V, Stone DL, Park JK, Callahan M, Frisch A, Cohen IJ, et al. Analysis and classification of 304 mutant alleles in patients with type 1 and type 3 Gaucher disease. Am J Hum Genet. 2000;66(6):1777-86

15. Matoth Y, Fried K. Chronic Gaucher's disease; clinical observations on 34 patients. Isr J Med Sci. 1965 Jul;1(4):521-30.

16. Figuera LE. Consenso Mexicano de Enfermedad de Gaucher. Rev Med Inst Mex Seguro Soc. 2010;48(2):167-86.

17. Beutler $E$, Saven A. Misuse of marrow examination in the diagnosis of Gaucher disease. Blood. 1990;76(3):646-8.

18. Shiran A, Brenner B, Laor A, Tatarsky I. Increased risk of cancer in patients with Gaucher disease. Cancer. 1993;72(1):219-24.

19. Mistry PK, Taddei T, vom Dahl S, Rosenbloom BE. Gaucher disease and malignancy: a model for cancer pathogenesis in an inborn error of metabolism. Crit Rev Oncog. 2013;18(3):235-46.

20. Pastores GM, Rosenbloom B, Weinreb N, Goker-Alpan O Grabowski G, Cohn GM, et al. A multicenter open-label treatment protocol (HGT-GCB-058) of velaglucerase alfa enzyme replacement therapy in patients with Gaucher disease type 1: safety and tolerability. Genet Med. 2014;16(5):359-66.

21. Lavaut K, Fernández R, Leal P, González A, Arencibia A Experiencia cubana en la terapia de reemplazo enzimático en la Enfermedad de Gaucher. Rev Cubana Hematol Inmunol Hemoter. 2015;31(2):187-94.

22. Hughes DA, Pastores GM. Haematological manifestation and complications of Gaucher disease. Curr Opin Hematol. 2013;20(1):41-7.

23. Llorca IL, Noguera OV. Enfermedad de Gaucher: a propósito de un caso. Rev Diagn Biol. 2002;51(3):100-4.

\section{Las ediciones anteriores de Acta Médica Peruana están disponibles en:}

\section{www.scielo.org.pe}

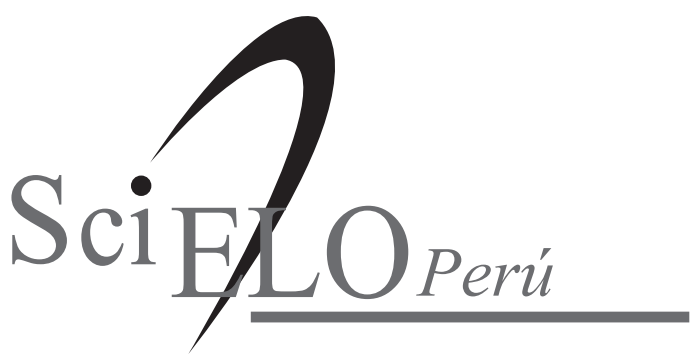

